Supporting Information

\title{
Bio-inspired Two-dimensional Structure with Asymmetric Wettability Barriers for Unidirectional and Long-Distance Gas Bubbles Delivery Underwater
}

Xiao Xiao, ${ }^{\text {\# }}$ Shukun Li, \& Xiandong Zhu, \& Xiao Xiao, ${ }^{+}$Chunhui Zhang, ${ }^{\mathcal{s}}$ Fengmin Jiang, ${ }^{\ddagger \star}$ Cunming $Y u,{ }^{* *}$ Lei Jiangts

${ }^{\dagger}$ Key Laboratory of Bio-inspired Smart Interfacial Science and Technology of Ministry of Education, School of Chemistry, Beihang University, Beijing 100191, China.

\#Department of Bioengineering, University of California, Los Angeles, Los Angeles, CA 90095, USA

§Laboratory of Bio-inspired Materials and Interface Sciences, Technical Institute of Physics and Chemistry, Chinese Academy of Sciences, Beijing 100190, China.

‡Beijing Institute of Technology, Beijing 100080, China. 


\section{Methods}

Preparation of Nepenthes alata-inspired 2D structures (NATS): The poly (methyl methacrylate) sheet (PMMA, $75 \mathrm{~mm} \times 25 \mathrm{~mm} \times 1 \mathrm{~mm}$, Available in Market) with good transparency, chemical stability, and accessible fabrication property, was selected as the substrate. Firstly, the polydimethylsiloxane (PDMS, Dow Corning, SYLGARD 184) prepolymer solution ( $1.0 \mathrm{~g}$ ) containing $10 \%$ curing agent was dissolved into $10 \mathrm{ml}$ of n-hexane (Beijing Chemical Plant, Analytically Pure) and stirred thoroughly. Then, immersing the PMMA sheet into the prepared solution for $30 \mathrm{~s}$ and place it in the air at room temperature for $10 \mathrm{~s}$. As such, the surface of the PMMA sheet will be coated with a thin PDMS layer.

Next, hydrophobic SiO2 nanoparticles (Aerosil R-972, average particle size $16 \mathrm{~nm}$, Evonik Degussa Co.) were uniformly deposited on the surface of PMMA sheet containing PDMS liquid. After heating it at 80 oC for 2 hours, the PDMS layer would be cured, which can tightly attach superhydrophobic nano-silica particles, and micro/nanostructures were constructed. The as-prepared sample was carefully rinsed with ample ethanol to remove the surplus superhydrophobic nano-silica particles and finally dried under nitrogen flow to achieve a superhydrophobic surface. 
Subsequently, under the assistant of the CO2-laser device with a cutting speed of 100 $\mathrm{mm} / \mathrm{s}$ and the output power of $27 \mathrm{~W}$ (LSC 30, HGLASER, China), which can lead to the melting and decomposing of PMMA, the superhydrophobic surface could be facilely cut into the expected shape within seconds. Finally, the NATS was successfully prepared. The other NATS, including the S-shaped surface and NATS electrode, could be fabricated with a similar process.

Instruments and Characterization: SEM images of the samples were obtained with a desktop scanning electron microscope (Phenom G2-Pro, Phenom World, Netherlands). The roughness of various surfaces, i.e., the superhydrophobic surface and the laser etching area, is obtained by a Confocal Laser Scanning Microscope (OLS4500, Olympus, Japan). The gas bubbles were injected on the NATS via a dual-channeltarget controlled syringe pump (CP-700TCI, Beijing slgo Medical Technology, China). The behaviors of the gas bubble on the NATS were recorded by using a high-speed camera (i-SPEED 3, Olympus, Japan). The water and bubble contact angles were measured using a video-based contact angle measuring device (OCA 40, Data-physics, Germany). The interaction force between the gas bubbles and the surface can be accessed by a high-sensitivity micro-electromechanical balance system (DCAT 11, Data-physics, Germany) with an underwater contact angle and adhesive force measuring liquid tank (self-built).

Finite element modeling: Finite element modeling was carried out using the commercial software COMSOL Multiphysics (COMSOL Inc., Burlington, MA). The bubble geometry during gas delivery was represented by a 3D model. In 
the simulations, the gas was considered as dry air, and the environment was considered as pure water. The bubble passing through the barriers was represented on a NATS with a width of $8 \mathrm{~mm}$. The flow rate of gas at the inlet with a diameter of $0.6 \mathrm{~mm}$ was set to $10^{-6} \mathrm{~m}^{3} / \mathrm{s}$. The peristaltic flow profile throughout the fluid geometry was calculated using the Navier-Stokes equation and level set method.

a

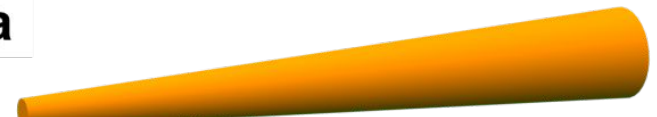

b

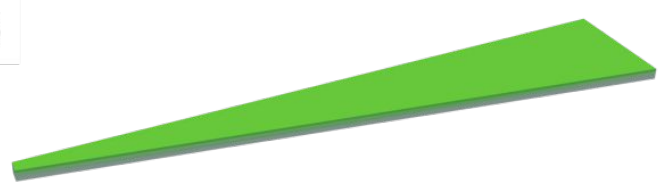

C

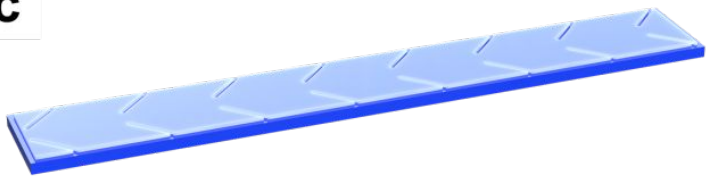

Figure S1. Emerging shape-gradient strategies to enable underwater gas bubble manipulation. a) The first reported cactus spine-inspired conical morphology is hard for mass production. ${ }^{1-4}$ Cone structures need special equipment, which is not economical for industrialization. b) Geometry-gradient surface is easily fabricated. ${ }^{5,6}$ Still, it has a poor performance in long-distance transportation similar to conical morphology. c) Our work addressed the intractable concerns by taking inspiration from Nepenthes alata, whose peristome surface consists of asymmetric microcavities with oriented edges inducing capillary rise. 


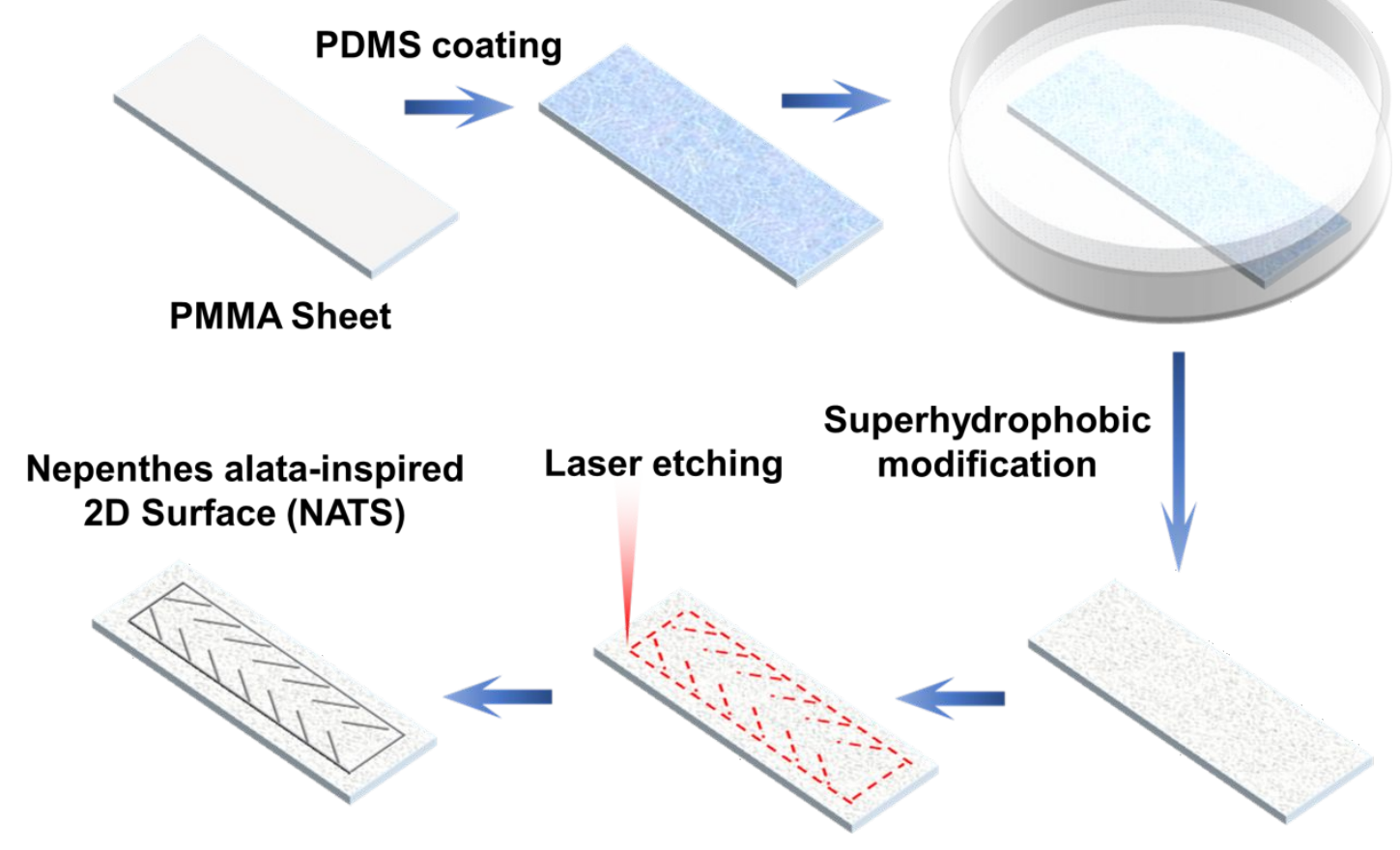

Figure S2. Schematic illustration of the preparation process of NATS on PMMA sheets. 


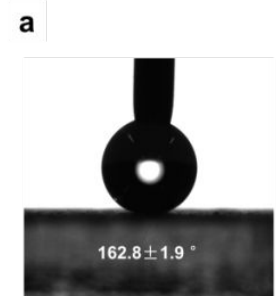

b

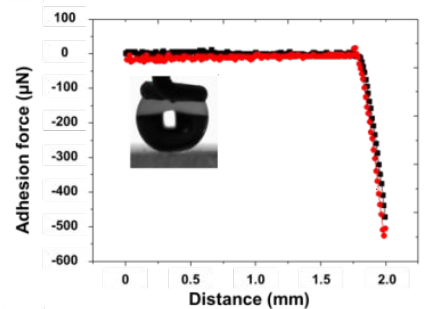

e

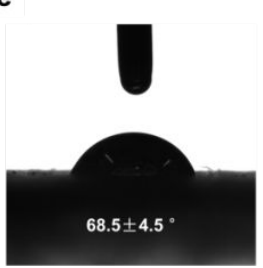

f

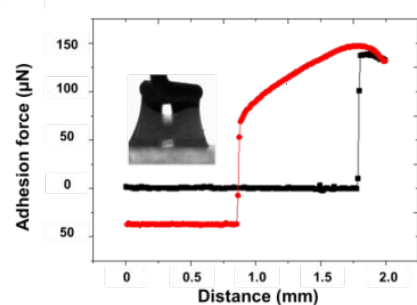

c d

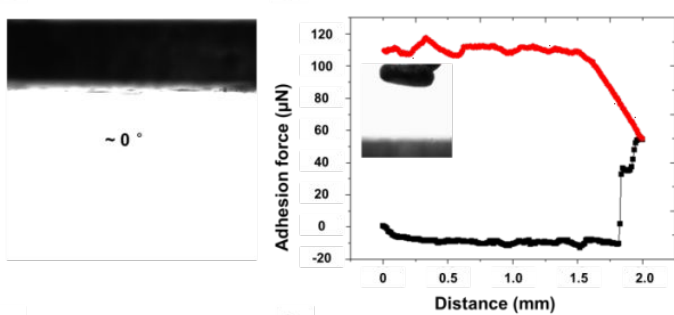

g

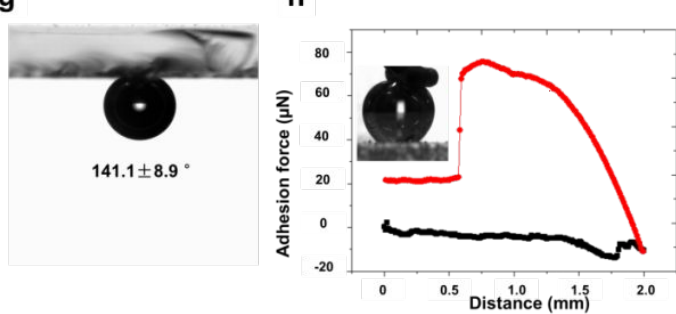

Figure S3. Wettability characterization of NATS. a, b, c, d) Water contact angle, droplet adhesion, bubble contact angle, bubble adhesion of superhydrophobic region. $\mathbf{e}, \mathbf{f}, \mathbf{g}, \mathbf{h})$ Water contact angle, droplet adhesion, bubble contact angle, bubble adhesion of laser etching region. 


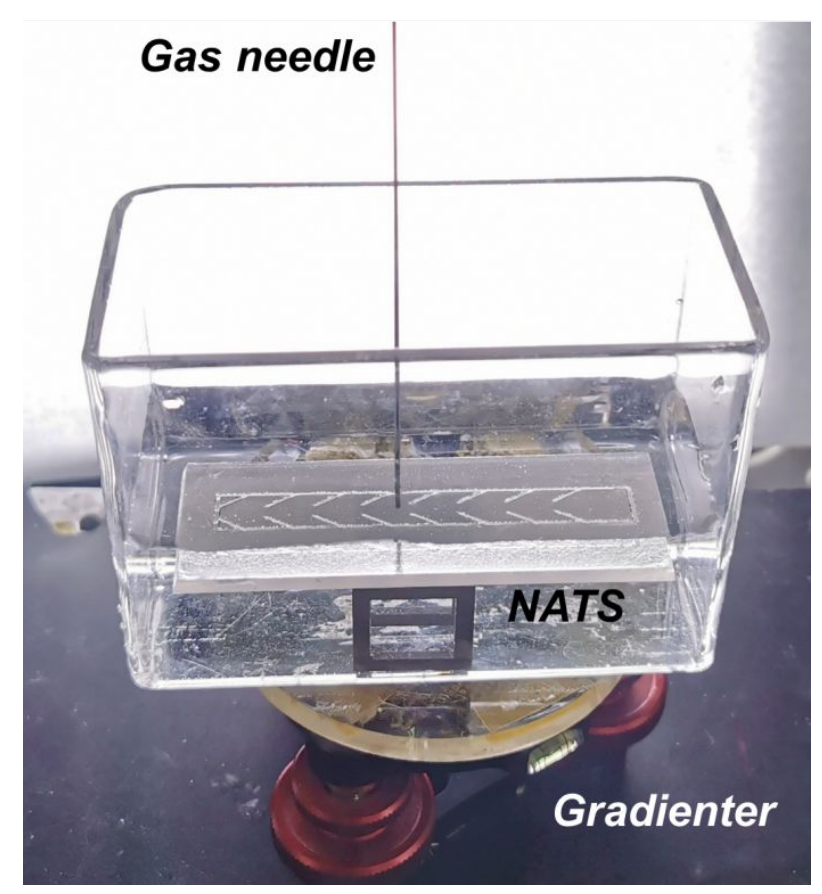

Figure S4. Setup of observing bubble transmission on NATS. A gas needle was set close to the NATS flake with a distance of $2 \mathrm{~mm}$ for in-situ gas releasing. The flow rate of gas at the inlet with a diameter of $0.6 \mathrm{~mm}$ was set to $10^{-6} \mathrm{~m}^{3} / \mathrm{s}$. The width of NATS is $8 \mathrm{~mm}$. 


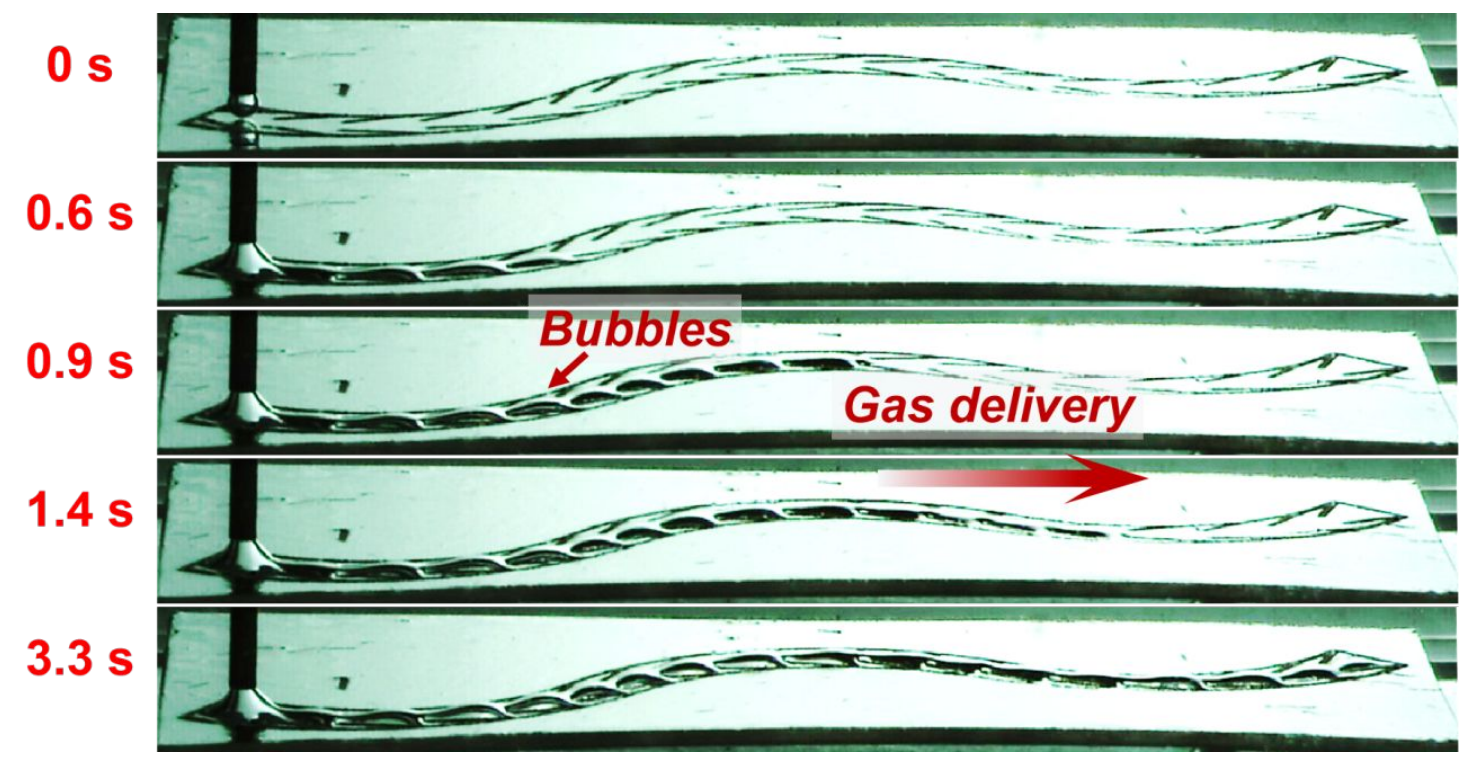

Figure S5. Optical images of gas bubbles transport on the S-shape NATS. 


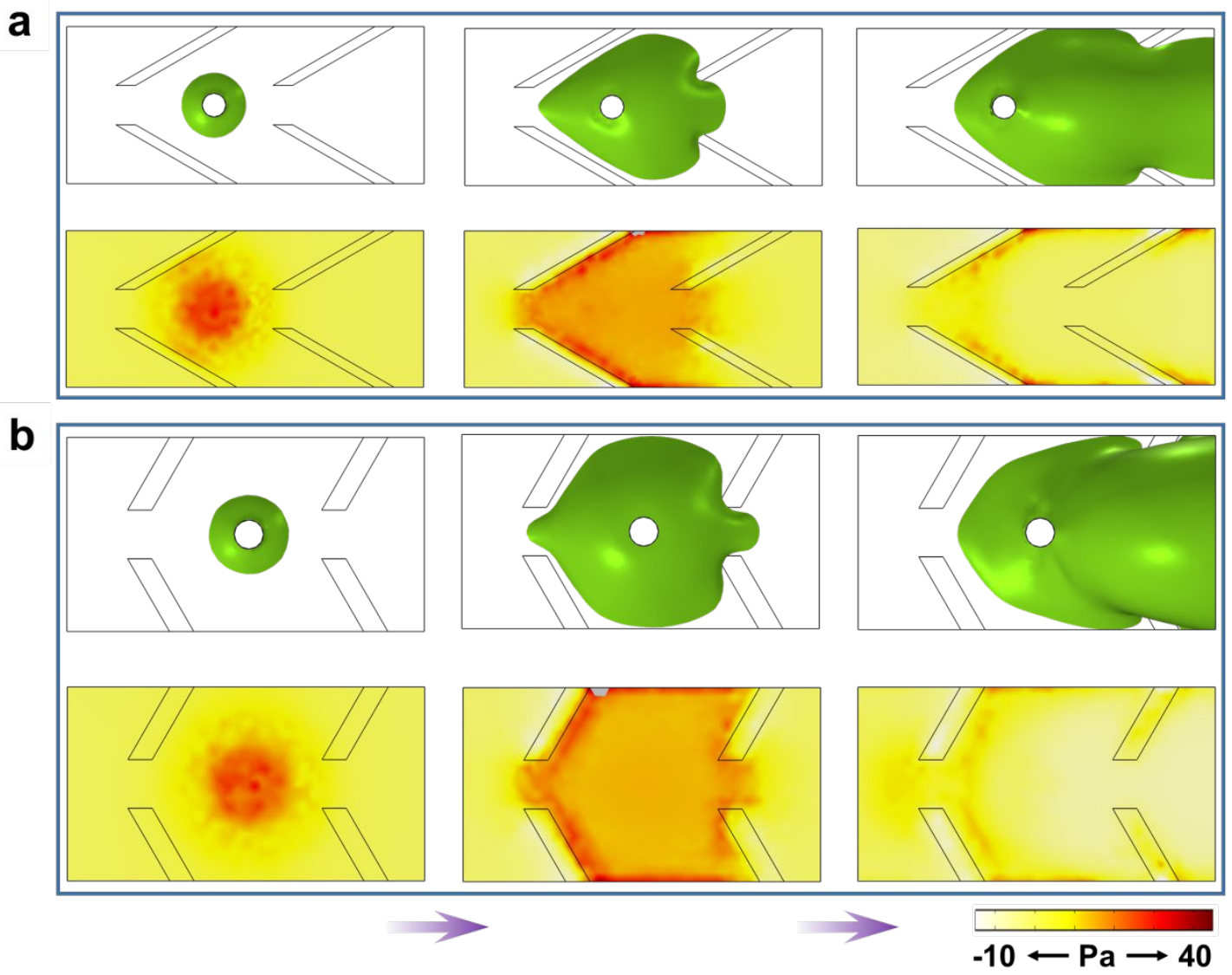

Figure S6. Schematical modeling and corresponding pressure of gas bubbles spreading on NATS with different angles of a) $30^{\circ}$, and b) $60^{\circ}$ unidirectionally by COMSOL Multiphysics. 


\section{REFERENCES}

(1) Yu, C. M.; Cao, M. Y.; Dong, Z. C.; Wang, J. M.; Li, K.; Jiang, L., Spontaneous and Directional Transportation of Gas Bubbles on Superhydrophobic Cones. Adv. Funct. Mater. 2016, 26 (19), 3236-3243.

(2) Yu, C. M.; Cao, M. Y.; Dong, Z. C.; Li, K.; Yu, C. L.; Wang, J. M.; Jiang, L., Aerophilic Electrode with Cone Shape for Continuous Generation and Efficient Collection of $\mathrm{H}_{2}$ Bubbles. Adv. Funct. Mater. 2016, 26 (37), 6830-6835.

(3) Xue, X. Z.; Yu, C. M.; Wang, J. M.; Jiang, L., Superhydrophobic Cones for Continuous Collection and Directional Transportation of $\mathrm{CO}_{2}$ Microbubbles in $\mathrm{CO}_{2}$ Supersaturated Solutions. ACS Nano 2016, 10 (12), 10887-10893.

(4) Xiao, X.; Zhang, C. H.; Ma, H. Y.; Zhang, Y. H.; Liu, G. L.; Cao, M. Y.; Yu, C. M.*; Jiang, L., Bioinspired Slippery Cone for Controllable Manipulation of Gas Bubbles in Low-Surface-Tension Environment. ACS Nano 2019, 13 (4), 4083-4090.

(5) Zhang, C. H.; Zhang, B.; Ma, H. Y.; Li, Z.; Xiao, X.; Zhang, Y. H.; Cui, X. Y.; Yu, C. M.*; Cao, M. Y.; Jiang, L., Bioinspired Pressure-Tolerant Asymmetric Slippery Surface for Continuous Self-Transport of Gas Bubbles in Aqueous Environment. ACS Nano 2018, 12 (2), 2048-2055.

(6) Ma, H. Y.; Cao, M. Y.; Zhang, C. H.; Bei, Z. L.; Li, K.; Yu, C. M.*; Jiang, L., Directional and Continuous Transport of Gas Bubbles on Superaerophilic Geometry-Gradient Surfaces in Aqueous Environments. Adv. Funct. Mater. 2018, 28 (7), 1705091. 
